\section{Bifidobacterium breve Sepsis in Child with High-Risk Acute Lymphoblastic Leukemia}

\section{Simona Lucija Avcin, Marko Pokorn, Lidija Kitanovski, Manica Mueller Premru, Janez Jazbec}

Author affiliations: University Medical Centre, Ljubljana, Slovenia (S.L. Avcin, M. Pokorn, L. Kitanovski, J. Jazbec); Institute for Microbiology and Immunology, Ljubljana (M.M. Premru)

\section{DOI: http://dx.doi.org/10.3201/eid2109.150097}

To the Editor: Patients with cancer often consume probiotics as part of their diet, although therapeutic use of probiotics is not recommended because of their potential invasiveness. In a recent review, 5 cases of probiotic treatment-related bacteremia were identified in oncology patients, although no cases of invasive Bifidobacterium spp. infection were included (1). We describe a case of $B$. breve sepsis in a child with Philadelphia chromosome-positive acute B-cell lymphoblastic leukemia.

The patient was a previously healthy 2-year-old boy who had no history of immunodeficiency and whose leukocyte counts had been within reference ranges during checkup visits before his diagnosis. After leukemia was diagnosed, chemotherapy was started (prednisone, vincristine, doxorubicin, and L-asparaginase). During the second week of treatment, the boy experienced abdominal discomfort and constipation. Two weeks later, his condition worsened; he refused food, his abdomen was distended, and he had colicky pain. Thickened intestinal wall and fecal masses were seen ultrasonographically. Twelve hours later he became hypotensive. Laboratory test results showed severe neutropenia and increased inflammatory markers (Figure). Two aerobic and anaerobic blood culture samples were collected from a central venous line (implantable venous access system) in a 30-minute span, and treatment with piperacillin/tazobactam, vancomycin, and gentamicin was empirically initiated according to local recommendations for pediatric febrile neutropenia with shock. Both anaerobic blood cultures were positive. Gram-positive, irregular rods with bifid and branching forms without spores grew anaerobically on blood agar with hemin and vitamin $\mathrm{K}$ after 48 hours of incubation and were identified as $B$. breve by matrix-assisted laser desorption/ionization time-of-flight mass spectrometry (Bruker Daltonics, Billerica, MA, USA). The bacteria were susceptible to penicillin (MIC $0.250 \mu \mathrm{g} / \mathrm{mL}$ ), ampicillin, amoxicillin/clavulanic acid, piperacillin/tazobactam (MIC $0.125 \mu \mathrm{g} / \mathrm{mL}$ ), imipenem, and clindamycin but not metronidazole. Gentamicin and vancomycin were discontinued, and piperacillin/tazobactam was replaced by penicillin (Figure). The patient stayed afebrile, and his neutropenia resolved. A blood culture taken on the eighth day of antimicrobial drug treatment was negative, and the central venous line was not replaced at that time. Bowel movement normalized and was maintained. We reviewed the ingredients of the food that the child received and documented the presence of Lactobacillus spp. and B. longum but not $B$. breve.

Some probiotics are part of the normal intestinal microbiota and rarely cause invasive infections (2). Although Bifidobacterium spp. is infrequently associated with infections ( $<5 \%$ of anaerobic isolates), it occasionally causes serious illness. On the rare occasions when it is isolated from patients with bloodstream infections, it is usually isolated along with other causative agents. The number of reported deaths associated with anaerobic nonsporulating gram-positive rods is low (3). In this patient, abdominal symptoms coincided with 2 blood cultures that yielded $B$. breve. We assume bacteria translocated through the distended colonic wall during chemotherapy-induced neutropenia and believe that the blood culture isolate was not a contaminant because it was isolated from 2 samples taken in a 30-min span. It is the practice at our institution not to take peripheral blood cultures simultaneously because doing so does not increase diagnostic accuracy.

We found 1 description in the literature of $B$. breve septicemia in a neonate with omphalocele who had received probiotic therapy (4). In a review of Bifidobacterium spp. isolates during 2000-2007 in 2 US hospitals, $B$. breve was isolated from blood culture from 3 adult patients (5). Two of these infections were associated with ileal resection or peritonitis and 1 with decubitus ulcers. No data on antimicrobial drug treatment were available. Bifidobacterium spp. sepsis was reported in an infant of extremely low birthweight 10 days after probiotic supplementation who recovered after antimicrobial drug therapy, although stenosis of the colon

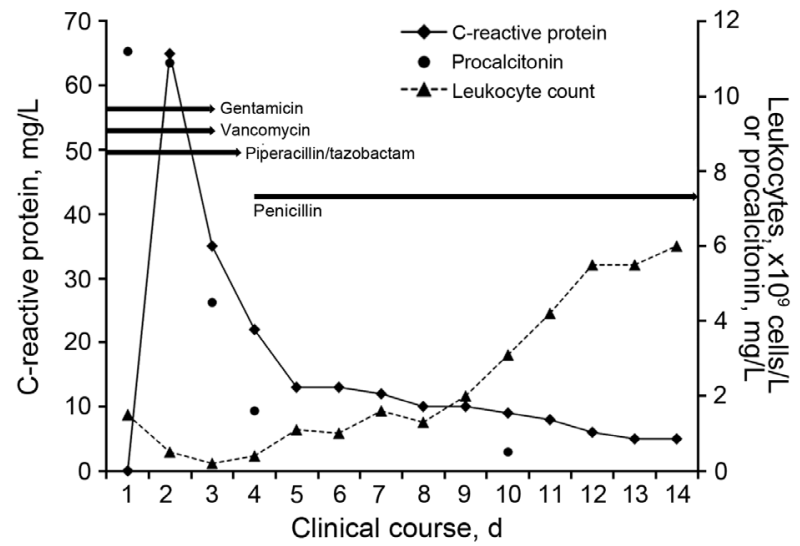

Figure. Schematic presentation of leukocyte count, C-reactive protein, and procalcitonin serum levels in clinical course of Bifidobacterium breve sepsis. Arrows indicate the name and duration of each antimicrobial drug treatment. 
developed 6 weeks later (6). Blood culture grew B. longum and $B$. infantis, which were probiotic strains. Apart from 1 case of sepsis caused by $B$. longum associated with acupuncture in a 19-year-old healthy patient (7), we did not find other reports of invasive Bifidobacterium spp. infections.

Because neutropenic episodes, even with bowel involvement, are common during treatment for cancer (8), no reason to promote therapeutic use of probiotics has been proven. Probiotics can cause substantial bacterial overgrowth when stimulating factors are present. In our opinion, avoiding fecal impaction is crucial for preventing colonic bacterial overgrowth and minimizes the chance that bacteria will translocate and cause invasive infection. $\mathrm{Nu}$ tritional recommendations for a neutropenic diet for children are still debated. The problem is not probiotic therapy but rather fermented food products to which small amounts of probiotics are added. After we reviewed the literature, we did not find enough data to safely recommend the use of these products in children receiving chemotherapy (9). Nevertheless, probiotic therapy is recommended for many immunocompromised patients, such as preterm infants and persons with chronic inflammatory bowel disease (10). We believe that this case of $B$. breve sepsis in an oncology patient underscores the invasive potential of probiotics.

\section{References}

1. Redman MG, Ward EJ, Phillips RS. The efficacy and safety of probiotics in people with cancer. Ann Oncol. 2014;25:1919-29. http://dx.doi.org/10.1093/annonc/mdu106

2. Bottacini F, Ventura M, van Sinderen D, O'Connell Motherway M. Diversity, ecology and intestinal function of bifidobacteria. Microb Cell Fact. 2014;13(Suppl 1):S4 http://dx.doi.org/ 10.1186/1475-2859-13-S1-S4.

3. de Vrese M, Schrezenmeir J. Probiotics, prebiotics, and synbiotics. Adv Biochem Eng Biotechnol. 2008;111:1-66. http://dx.doi.org/10.1007/10_2008_097.

4. Ohishi A, Takahashi S, Ito Y, Ohishi Y, Tsukamoto K, Nanba Y, et al. Bifidobacterium septicemia associated with postoperative probiotic therapy in a neonate with omphalocele. J Pediatr. 2010;156:679-81. http://dx.doi.org/10.1016/j.jpeds.2009.11.041

5. Mahlen SD. Clarridge JE 3rd. Site and clinical significance of Alloscardovia omnicolens and Bifidobacterium species isolated in the clinical laboratory. J Clin Microbiol. 2009;47:3289-93. http://dx.doi.org/10.1128/JCM.00555-09

6. Jenke A, Ruf EM, Hoppe T, Heldmann M, Wirth S. Bifidobacterium septicaemia in an extremely low-birthweight infant under probiotic therapy. Arch Dis Child Fetal Neonatal Ed. 2012;97:F217-8.

7. Ha GY, Yang CH, Kim H, Chong Y. Case of sepsis caused by Bifidobacterium longum. J Clin Microbiol. 1999;37:1227-8.

8. Rizzatti M, Brandalise SR, de Azevedo AC, Pinheiro VR, Aguir Sdos S. Neutropenic enterocolitis in children and young adults with cancer: prognostic value of clinical and image findings. Pediatr Hematol Oncol. 2010;27:462-70. http://dx.doi.org/10.3109/ 08880018.2010 .489934

9. Moody K, Charlson ME, Finlay J. The neutropenic diet: what's the evidence? J Pediatr Hematol Oncol. 2002;24:717-21. http://dx.doi.org/10.1097/00043426-200212000-00007

10. Floch MH. Recommendations for probiotic use in humansa 2014 update. Pharmaceuticals (Basel). 2014;7:999-1007. http://dx.doi.org/10.3390/ph7100999
Address for correspondence: Simona Avcin, University Children's Hospital, University Medical Centre, Bohoriceva 20, Ljubljana, Slovenia; email: simona.avcin@kclj.si

\section{Filovirus RNA in Fruit Bats, China}

\section{Biao He, ${ }^{1}$ Yun Feng, ${ }^{1}$ Hailin Zhang, ${ }^{1}$ Lin $\mathrm{Xu}$, Weihong Yang, Yuzhen Zhang, Xingyu Li, Changchun Tu}

Author affiliations: Academy of Military Medical Sciences, Jilin, China (B. He, L. Xu, X. Li, C. Tu); Yunnan Institute of Endemic Diseases Control and Prevention, Dali, China (Y. Feng, H. Zhang, W. Yang, Y. Zhang); Jiangsu Co-innovation Center for Prevention and Control of Important Animal Infectious Diseases and Zoonoses, Yangzhou University, Yangzhou, China (C. Tu)

DOI: http://dx.doi.org/10.3201/eid2109.150260

To the Editor: Filovirus-associated diseases, particularly those caused by Ebola and Marburg viruses, represent major threats to human health worldwide because they have extremely high death rates and antiviral therapies or vaccines against them are not available (1). Members of the family Filoviridae are classified into 3 genera: Marburgvirus, Ebolavirus, and the recently approved Cuevavirus $(2,3)$. Marburg virus (MARV) and Ebola virus (EBOV) were initially isolated in Africa, but other filoviruses have been identified on other continents. The initial Cuevavirus, Lloviu virus (LLOV), was identified in Europe (Spain) (3), and Ebola-Reston virus has been found in pigs in Asia (the Philippines) (4).

Bats are natural reservoirs for filoviruses (5). Viral isolation and serologic studies indicate that filovirus infections have occurred in various bat species in central Africa countries (6), the Philippines (7), China (8), and Bangladesh (9). However, identification of these viruses in bats has been difficult; although isolates of MARV have been obtained (G) and the genome of LLOV has been fully sequenced (3), very short sequences of EBOV have been obtained from bats, and only in Africa (5). Reports of molecular detection or isolation of filoviruses in bats in Asia are lacking. We conducted a study to investigate the presence of filoviruses in bats in China.

In June 2013, twenty-nine apparently healthy Rousettus leschenaultia fruit bats were captured in Yunnan Province, China. All bats were humanely killed, and their intestines, lungs, livers, and brains were collected and subjected to viral metagenomic analysis by a previously described method (10). As a result, we obtained and reassembled de novo

${ }^{1}$ These authors contributed equally to this article. 